\title{
GROWTH CONDITIONS FOR ANALYTIC FUNCTIONS IN UNBOUNDED OPEN SETS
}

\author{
AIMO HINKKANEN \\ Dedicated to Professor Chung-Chun Yang on the occasion of his $65^{\text {th }}$ birthday
}

\section{IntRoduction AND STATEMENT OF RESUlts}

Let $G$ be a non-empty open set in the complex plane $\mathbb{C}$ with at least two finite boundary points. We define the boundary $\partial G$ and closure $\bar{G}$ of $G$ as subsets of $\mathbb{C}$, hence not containing the point at infinity even if $G$ were unbounded. If there is $R>0$ such that $G$ contains the set $\{z \in \mathbb{C}:|z|>R\}$, we say for brevity that $G$ contains a neighbourhood of infinity, even though it might be more accurate to say that $G$ contains a punctured neighbourhood of infinity. Let $f: \bar{G} \rightarrow \mathbb{C}$ be a continuous function that is analytic in $G$. We refine earlier majorisation results for $f$ when $G$ is unbounded.

When discussing upper bounds for the modulus of continuity of $f$, it has turned out to be natural and even necessary (compare [15]) to limit oneself to upper bounds that have certain regularity properties. We say that a non-decreasing non-negative function $\mu$ defined for $t \geq 0$ is a majorant if

$$
\mu(2 t) \leq 2 \mu(t)
$$

for all $t \geq 0$.

In two recent papers ([13], [12]), the author has obtained results of the following kind. Suppose that

$$
\left|f\left(z_{1}\right)-f\left(z_{2}\right)\right| \leq \mu\left(\left|z_{1}-z_{2}\right|\right)
$$

for a fixed $z_{1} \in \partial G$ and for all $z_{2} \in \partial G$. Then (under suitable extra conditions if $G$ is unbounded)

$$
\left|f\left(z_{1}\right)-f\left(z_{2}\right)\right| \leq C \mu\left(\left|z_{1}-z_{2}\right|\right)
$$

where $C$ is an absolute constant. The novelty of such a result is that earlier, estimates of this type for an arbitrary majorant, as opposed to special majorants such as $\mu(t)=M t^{\alpha}$, where $M>0$ and $0<\alpha \leq 1$,

2000 Mathematics Subject Classification. Primary 30C80.

This material is based upon work supported by the National Science Foundation under Grant No. 0457291. 
had been obtained only if $G$ is a simply [16] or doubly [9] connected domain.

The author obtained $C=3456$, which clearly must be far from best possible. This was proved in [13], Theorem 1.11 when every component of $G$ is bounded, and in [12], Theorem 1.2 under the condition that $f(z)=o(z)$ as $z \rightarrow \infty$ in each unbounded component of $G$. On the other hand, the author ([10], Theorem 1, p. 41) has proved that when $G$ is the unit disk $\{z \in \mathbb{C}:|z|<1\}$, then the best possible constant $C$ in this result is

$$
C=\exp \left\{\frac{\log 4}{\pi} \sum_{n=0}^{\infty} \arctan 2^{-n}\right\} \approx 2.158173 .
$$

In this paper we prove the following result.

Theorem 1. Let $G$ be a non-empty open set in the complex plane $\mathbb{C}$ with at least two finite boundary points. Let $f: \bar{G} \rightarrow \mathbb{C}$ be a continuous function that is analytic in $G$. Let $\mu$ be a majorant. Suppose that

$$
\left|f\left(z_{1}\right)-f\left(z_{2}\right)\right| \leq \mu\left(\left|z_{1}-z_{2}\right|\right)
$$

for a fixed $z_{1} \in \partial G$ and for all $z_{2} \in \partial G$. Suppose that for each unbounded component $D$ of $G$, if any, there is a positive number $q$ such that

$$
f(z)=O\left(|z|^{q}\right) \quad \text { as } z \rightarrow \infty \text { in } D .
$$

Then one of the following holds:

(i) For all $z_{2} \in G$ we have

$$
\left|f\left(z_{1}\right)-f\left(z_{2}\right)\right| \leq C \mu\left(\left|z_{1}-z_{2}\right|\right)
$$

where $C=3456$.

(ii) The set $G$ contains a neighbourhood of infinity, so that $G$ has exactly one unbounded component, and $f$ has a pole at infinity.

Furthermore, if (1.5) holds for all $z_{1}, z_{2} \in \partial G$, then (1.7) holds for all $z_{1}, z_{2} \in \bar{G}$ with $C=3456$ unless $G$ contains a neighbourhood of infinity, so that $G$ has exactly one unbounded component, and $f$ has a pole at infinity.

The only case when both (i) and (ii) of Theorem 1 may be valid is when $G$ contains a neighbourhood of infinity and $f$ has a simple pole at infinity. In such a case, for (1.5) to hold for all $z_{2} \in \partial G$ (or even for all $z_{1}, z_{2} \in \partial G$ ), it only matters what the values of $\mu(t)$ are for $0 \leq t \leq \operatorname{diam}(\partial G)$ where $\operatorname{diam}(\partial G)$ denotes the Euclidean diameter of $\partial G$. Just for $\mu$ to be a majorant, it would be admissible to have $\mu(t)=\mu(\operatorname{diam}(\partial G))$ for all $t \geq \operatorname{diam}(\partial G)$, and in that case (i) could 
not hold for all $z_{2} \in G$ when $f$ has even just a simple pole at infinity. On the other hand, since $\mu(t)$ may grow like a constant multiple of $t$ as $t \rightarrow \infty$, there remains the possibility that (i) may hold if $f$ has a simple pole at infinity. We note that if $\mu(t)=M t$ for some positive constant $M$, then by [4], Theorem 1, p. 243, (i) holds with $C=1$ when $f$ has a simple pole at infinity.

In order to deal with one of the cases arising in the proof of Theorem 1, we will prove the following result dealing with the growth of analytic functions in unbounded open sets. Perhaps this result is known, but we have not been able to find it in the literature in this generality.

Theorem 2. Let $G$ be an unbounded open set in the complex plane $\mathbb{C}$ such that its boundary $\partial G$ is also unbounded. Let $f: \bar{G} \rightarrow \mathbb{C}$ be a continuous function that is analytic in $G$. Suppose that for each unbounded component $D$ of $G$, if any, there is a positive number $q$ such that (1.6) holds. Then

$$
\limsup _{z \rightarrow \infty, z \in \bar{G}} \frac{|f(z)|}{|z|}=\limsup _{z \rightarrow \infty, z \in \partial G} \frac{|f(z)|}{|z|} .
$$

Remark 1. Since clearly

$$
\limsup _{z \rightarrow \infty, z \in \bar{G}} \frac{|f(z)|}{|z|} \geq \limsup _{z \rightarrow \infty, z \in \partial G} \frac{|f(z)|}{|z|},
$$

(1.8) is equivalent to

$$
\limsup _{z \rightarrow \infty, z \in \bar{G}} \frac{|f(z)|}{|z|} \leq \limsup _{z \rightarrow \infty, z \in \partial G} \frac{|f(z)|}{|z|} .
$$

Thus to prove Theorem 2, it suffices to prove (1.9).

Remark 2. It follows that if (1.6) holds and if

$$
\lim _{z \rightarrow \infty, z \in \partial G} \frac{|f(z)|}{|z|}=0
$$

then

$$
\lim _{z \rightarrow \infty, z \in \bar{G}} \frac{|f(z)|}{|z|}=0 .
$$

Note that Theorem 2 allows for the possibility that all components of $G$ are bounded; the proof that we give works in the same way whether or not that is the case. In this case, however, the result follows from the maximum modulus principle possibly combined with an argument involving harmonic measure, applied to each component of $G$ separately.

We have formulated (1.9) in terms of the quantity $f(z) / z$ since this is what we need when we apply Theorem 2 in the proof of Theorem 1 . 
Obviously the inequality obtained by replacing $f(z) / z$ by $f(z)$ on both sides of (1.9) is also valid.

One may view Theorem 2 as a refinement of a result of Wolfgang Fuchs [3]. For simplicity, suppose that $0 \notin \bar{G}$, and pick a number $A$ slightly larger than $\lim \sup _{z \rightarrow \infty, z \in \partial G}|f(z) / z|$, which we may assume to be finite. Applied to the function $F(z)=f(z) /(A z)$, Fuchs's theorem states that one of the following must hold if $|F(z)| \leq 1$ for all $z \in \partial G$ : (i) $|F(z)| \leq 1$ for all $z \in G$; (ii) $G$ contains a neighbourhood of infinity and $F$ has a pole at infinity; or (iii) $\lim _{r \rightarrow \infty} \log M(r) / \log r=\infty$, where

$$
M(r)=\sup \{|F(z)|:|z|=r, z \in G\} .
$$

In Theorem 2 we do not have the assumption that $|F(z)| \leq 1$ for all $z \in \partial G$ but only for all $z \in \partial G$ such that $|z|$ is sufficiently large. On the other hand, we have the additional assumption (if compared to Fuchs's theorem) that $\partial G$ is unbounded, preventing $G$ from containing a neighbourhood of infinity, and the assumption (1.6) guarding against (iii), at least in the weaker sense of preventing it in each unbounded component of $G$ separately.

One might think that (1.9) should be true if $\partial G$ is sufficiently thick close to infinity. If, hypothetically, (1.9) fails, then it turns out firstly that $\partial G$ is indeed thin close to infinity and then that $G$ contains a neighbourhood of infinity, which contradicts the assumption that $\partial G$ is unbounded. This, roughly speaking, is the idea behind the proof of Theorem 2. These ideas go back to [4], on whose techniques we will heavily rely.

\section{Previous Results}

We have already made some comments on previous results of the type of Theorem 1. A survey has also been given in the paper [13] by the author. For completeness, we give more background particularly concerning results for unbounded open sets.

Let us first point out that there are two rather different problems involved, and also two different types of methods. One is based on potential theory alone and in particular only on the fact that if $f$ is analytic then $\log |f|$ is subharmonic. The other uses also the argument of $f$ and hence makes more complete use of the assumption that $f$ is analytic.

2.1. Hölder continuous functions. One particularly interesting majorant is $\mu(t)=M t^{\alpha}$, where $M>0$ and $0<\alpha \leq 1$. This majorant arises in the study of Hölder continuous functions $f$. For the early history of the results obtained for this majorant we refer to [13], and 
here we only quote the two strongest known results. The first is from [4], Theorem 1, p. 243.

Theorem 3. Let $G$ be a non-empty open set in $\mathbb{C}$ such that $\partial G$ contains at least two finite points, and let $f$ be continuous in $\bar{G}$ and analytic in $G$. Suppose that $0 \leq \alpha \leq 1$ and $M>0$, and that

$$
\left|f\left(z_{1}\right)-f\left(z_{2}\right)\right| \leq M\left|z_{1}-z_{2}\right|^{\alpha}
$$

whenever $z_{1}, z_{2} \in \partial G$. If $G$ is unbounded, suppose further that

$$
f(z)=o(|z|)
$$

as $z \rightarrow \infty$ in each unbounded component of $G$ if $\alpha<1$, and that

$$
f(z)=o\left(|z|^{2}\right)
$$

as $z \rightarrow \infty$ in each unbounded component of $G$ if $\alpha=1$. Then (2.1) holds for every pair of points $z_{1}, z_{2} \in \bar{G}$.

Further, if (2.1) holds for a fixed $z_{1} \in \partial G$ and for all $z_{2} \in \partial G$, then (2.1) also holds for this $z_{1}$ and for all $z_{2} \in \bar{G}$.

As pointed out in [4], taking $G=\{z:|z|>1\}$ and $f(z)=z$ and $f(z)=z^{2}$, respectively, we see that we cannot replace $o$ by $O$ in $(2.2)$ and (2.3).

The assumption that $\partial G$ contains at least two finite points is necessary, for otherwise the assumption (2.1) of Theorem 3 is vacuous while the conclusion does not always hold (see case (ii) in Theorem 4).

However, we may omit the assumption that $\partial G$ contains at least two finite points provided that we have a different assumption taking (in the special case when $G$ has only one finite boundary point) the role of (2.1) of Theorem 3. The following result was obtained by Tamrazov in [18], Theorem $1^{\alpha}$, p. 194. In this result, the exponent $\alpha$ is merely assumed to be a real number, not necessarily in $[0,1]$ or even positive. If we were dealing with a conclusion where we wish (2.1) to hold for all $z_{1}, z_{2} \in G$, then, if $f$ is not constant, we could hope to have such a conclusion only if $\alpha \leq 1$, and the use of a negative $\alpha$ in such a connection would be unusual. But in Theorem 4 below, we estimate $|f(z)|$ for $z \in G$, and not the moduli of differences $f\left(z_{1}\right)-f\left(z_{2}\right)$ for $z_{1}, z_{2} \in G$. This allowed Tamrazov to use any real exponent $\alpha$. If we wanted to apply Theorem 4 to estimate $\left|f\left(z_{1}\right)-f\left(z_{2}\right)\right|$ for a fixed $z_{1} \in \partial G$ and for all $z_{2} \in G$, we could do so by applying Theorem 4 to $f(z)-f\left(z_{1}\right)$ instead of $f$. As Theorem 8 to be given in Section 3 and its use in the proof of Theorem 1 show, it can be useful to have a way of estimating only $|f|$ even if one's ultimate aim were to find an upper bound for the quantity $\left|f\left(z_{1}\right)-f\left(z_{2}\right)\right|$. 
Theorem 4. Suppose that $z_{1} \in \mathbb{C}$, and let $G$ be a non-empty open set with $G \subset \mathbb{C} \backslash\left\{z_{1}\right\}$. Let $\alpha$ be a real constant, and let $\beta$ be a constant with $\beta \geq 0$. Let $f$ be a continuous complex-valued function in $\bar{G} \backslash\left\{z_{1}\right\}$ such that $f$ is analytic in $G$. Suppose that for each unbounded component $D$ of $G$, if any, there is a positive number $q$ such that (1.6) holds. Suppose that for each component $D$ of $G$ with $z_{1} \in \partial D$, if any, there is a positive number q such that

$$
|f(z)|=O\left(\left|z-z_{1}\right|^{-q}\right) \quad \text { as } z \rightarrow z_{1} \text { in } D .
$$

Suppose that

$$
|f(z)| \leq \beta\left|z-z_{1}\right|^{\alpha} \quad \text { for all } z \in \partial G \backslash\left\{z_{1}\right\} .
$$

Let $\alpha_{*}$ be the largest integer and let $\alpha^{*}$ be the smallest integer such that $\alpha_{*}<\alpha<\alpha^{*}$. If $z_{1}$ is an isolated boundary point of $G$, suppose further that

$$
|f(z)|=o\left(\left|z-z_{1}\right|^{\alpha_{*}}\right) \quad \text { as } z \rightarrow z_{1} .
$$

If $G$ contains a neighbourhood of infinity, suppose also that

$$
|f(z)|=o\left(|z|^{\alpha^{*}}\right) \quad \text { as } z \rightarrow \infty .
$$

Then exactly one of the following two possibilities holds:

(i) we have

$$
|f(z)| \leq \beta\left|z-z_{1}\right|^{\alpha} \quad \text { for all } z \in \bar{G} \backslash\left\{z_{1}\right\},
$$

or

(ii) we have $G=\mathbb{C} \backslash\left\{z_{1}\right\}$, $\alpha$ is an integer, and $f(z)=c\left(z-z_{1}\right)^{\alpha}$, where $c$ is a complex constant with $|c|>\beta$.

The condition $|c|>\beta$ is present in the conclusion (ii) of Theorem 4 only to make the possibilities (i) and (ii) mutually exclusive.

In Theorem 4 , even if we were to apply it to $f(z)-f\left(z_{1}\right)$ instead of $f$, for a fixed $z_{1} \in \partial G$ and for a given open set $G$, we would have to keep $z_{1}$ fixed also in the conclusion, so that we would not immediately get an estimate for $f(z)-f(w)$ for all $z, w \in G$. Concerning this problem, Tamrazov proved the following result in [18], Theorem $2^{\alpha}$, p. 195.

Theorem 5. Let $G$ be a non-empty open set in $\mathbb{C}$. Let $\alpha$ be a real constant, let $\alpha^{*}$ be defined as in Theorem 4 , and let $\beta$ be a constant with $\beta \geq 0$. Let $f: \bar{G} \rightarrow \mathbb{C}$ be continuous in $\bar{G}$ and analytic in $G$. Suppose that for each unbounded component $D$ of $G$, if any, there is a positive number q such that (1.6) holds.

If $G$ contains a neighbourhood of infinity, suppose also that $\alpha \geq 0$ and either that (2.7) is satisfied or that $f$ extends to a continuous function of $\bar{G} \cup\{\infty\}$ into $\mathbb{C}$. 
Suppose that

$$
\left|f\left(z_{1}\right)-f\left(z_{2}\right)\right| \leq \beta\left|z_{1}-z_{2}\right|^{\alpha} \quad \text { for all } z_{1}, z_{2} \in \partial G .
$$

Then the following statements are true:

(a) we have

$$
\left|f\left(z_{1}\right)-f\left(z_{2}\right)\right| \leq \beta\left|z_{1}-z_{2}\right|^{\alpha}
$$

whenever $z_{1}$ is a non-isolated boundary point of $G$ and $z_{2} \in \bar{G}$;

(b) if $f$ extends to a continuous function of $\bar{G} \cup\{\infty\}$ into $\mathbb{C}$ then (2.10) holds for all $z_{1}, z_{2} \in \bar{G}$; and

(c) if $\alpha \in(-\infty, 1]$, then exactly one of the following possibilities occurs:

(c1) (2.10) holds for all $z_{1}, z_{2} \in \bar{G}$;

(c2) $\alpha=1, \mathbb{C} \backslash G$ contains at most one point, and $f(z)=c z+b$ where $c$ and $b$ are complex constants with $|c|>\beta$.

Again, the condition $|c|>\beta$ appears in (c2) to make (c1) and (c2) mutually exclusive. Above, in cases (b) and (c1) we find sufficient conditions for (2.10) to hold for all $z_{1}, z_{2} \in \bar{G}$.

2.2. Bilogarithmically concave upper bounds. In order to obtain sharp results using subharmonicity, the function $\mu$ appearing on the right hand side of (1.2) and (1.3) and other similar inequalities, must have a suitable property causing a certain function to be subharmonic. The condition on $\mu$ required for the function

$$
\log \left|f(z)-f\left(z_{1}\right)\right|-\log \mu\left(\left|z-z_{1}\right|\right)
$$

to be a subharmonic function of $z$ in $G$ for each fixed $z_{1} \in \partial G$ is precisely that $\log \mu\left(e^{t}\right)$ must be a concave function of $t$ for real $t$. Tamrazov [17] has called such functions $\mu$ bilogarithmically concave. Since it is not clear that such a function $\mu$ needs to satisfy the condition (1.1), which we have taken as the defining property of a majorant, we shall not call such functions $\mu$ "bilogarithmically concave majorants".

The author proved the following result in [8], Theorem 1, p. 247.

Theorem 6. Let $G$ be an open set in $\mathbb{C}$ such that $\partial G$ contains at least two finite points, and let $f$ be continuous in $\bar{G}$ and analytic in $G$. Let $\mu$ be a non-decreasing non-negative function defined for $t \geq 0$ such that $\log \mu\left(e^{t}\right)$ is a concave function of $t$ for real $t$, and

$$
B \equiv \lim _{t \rightarrow 0+} \frac{\log \mu(t)}{\log t} \leq 1
$$


Set

$$
A=\lim _{t \rightarrow \infty} \frac{\log \mu(t)}{\log t} \leq B \leq 1,
$$

and assume that as $z \rightarrow \infty$ in any unbounded component of $G$, we have

$$
f(z)=o(|z|)
$$

if $A<1$, and

$$
f(z)=o\left(|z|^{2}\right)
$$

if $A=1$. Suppose that

$$
\left|f\left(z_{1}\right)-f\left(z_{2}\right)\right| \leq \mu\left(\left|z_{1}-z_{2}\right|\right)
$$

whenever $z_{1}, z_{2} \in \partial G$. Then (2.15) holds for every pair of points $z_{1}, z_{2} \in \bar{G}$.

Further, if (2.15) holds for a fixed $z_{1} \in \partial G$ and for all $z_{2} \in \partial G$, then (2.15) also holds for this $z_{1}$ and for all $z_{2} \in \bar{G}$.

If, instead of (2.13) or (2.14), we only have, for some fixed $q>$ 0 that depends only on $f$ and $G$, that $f(z)=o\left(|z|^{q}\right)$ as $z \rightarrow \infty$ in any unbounded component of $G$, and if (2.15) holds as assumed but the conclusion of Theorem 6 fails, then $G$ contains a neighbourhood of infinity, and $f$ has a pole at infinity.

Regarding the condition of Theorem 6 that for some fixed $q>0$ that depends only on $f$ and $G$, we have $f(z)=o\left(|z|^{q}\right)$ as $z \rightarrow \infty$ in any unbounded component of $G$, we may note that in Theorem 1 , this condition has been relaxed in such a way that $q$ may now depend also on the unbounded component of $G$ being considered and no longer needs to be the same for all such components. This can theoretically make a difference if $G$ has infinitely many unbounded components. Of course, the conclusion of Theorem 1 shows that in retrospect, either $G$ has only one unbounded component and hence there is only one number $q$ to consider, or we may take $q=1$ for all unbounded components of $G$ since it follows from (1.1) (see also (3.8) below) that $\mu(t)=O(t)$ as $t \rightarrow \infty$. This relaxation of the condition on $q$ is present already in Theorems 4 and 5, and in Theorem 7 below, all of which appeared in Tamrazov's paper [18].

Several contributions to the majorisation problem when $\mu$ is bilogarithmically concave have been made by Tamrazov. In [18], Theorem 1, p. 196, he obtained the following result. He considered also the possibility that a majorant $\mu$ is bilogarithmically concave only on an interval of the real axis while $\mu$ vanishes identically outside this interval. For 
simplicity, we state the result only in the case when $\mu$ is bilogarithmically concave everywhere, so that we can have $\mu(t)=0$ at most when $t=0$. For the most general formulation, we refer to [18].

Theorem 7. Suppose that $z_{1} \in \mathbb{C}$, and let $G$ be a non-empty open set with $G \subset \mathbb{C} \backslash\left\{z_{1}\right\}$. Let $\mu$ be a positive function defined for $t>0$ such that $\log \mu\left(e^{t}\right)$ is a concave function of $t$ for real $t$. Let $f$ be a continuous complex-valued function in $\bar{G} \backslash\left\{z_{1}\right\}$ such that $f$ is analytic in $G$. Suppose that for each unbounded component $D$ of $G$, if any, there is a positive number q such that (1.6) holds. Suppose that for each component $D$ of $G$ with $z_{1} \in \partial D$, if any, there is a positive number $q$ such that (2.4) holds. Suppose that

$$
|f(z)| \leq \mu\left(\left|z-z_{1}\right|\right) \quad \text { for all } z \in \partial G \backslash\left\{z_{1}\right\} .
$$

Let the numbers $A$ and $B$ be defined as in (2.12) and (2.11), so that $B>-\infty$ and $A<+\infty$. If $z_{1}$ is an isolated boundary point of $G$, suppose that $B<+\infty$ and that

$$
|f(z)|=o\left(\left|z-z_{1}\right|^{m_{0}-1}\right) \quad \text { as } z \rightarrow z_{1},
$$

where the integer $m_{0}$ satisfies $m_{0}-1<B \leq m_{0}$.

If $G$ contains a neighbourhood of infinity, suppose that $A>-\infty$ and that

$$
|f(z)|=o\left(|z|^{m_{\infty}+1}\right) \quad \text { as } z \rightarrow \infty,
$$

where the integer $m_{\infty}$ satisfies $m_{\infty} \leq A<m_{\infty}+1$.

Then exactly one of the following two possibilities holds:

(i) we have

$$
|f(z)| \leq \mu\left(\left|z-z_{1}\right|\right) \quad \text { for all } z \in G
$$

or

(ii) we have $G=\mathbb{C} \backslash\left\{z_{1}\right\}, \mu(t)=\beta t^{m}$, where $m=m_{0}=m_{\infty}$ is an integer and $\beta \geq 0$, and $f(z)=c\left(z-z_{1}\right)^{m}$, where $c$ is a complex constant with $|c|>\beta$.

The methods used by Tamrazov in the proof of Theorems 4, 5, and 7 as well as the methods used by the author to obtain Theorem 6 are to a large extent based on generalisations of the techniques used in [4].

Tamrazov proved in [16], Theorem 9.3, p. 167, that the condition that $\mu$ is bilogarithmically concave is necessary for a result like Theorem 7 to hold (that is, for us to have the smallest possible constant $C=1$ in (1.3)) even if we limit $G$ to be an arbitrary bounded Jordan domain.

In [19], Tamrazov proved extensions of Theorem 7 to holomorphic functions $f: G \rightarrow \mathbb{C}^{k}$, where $G$ is an open set in $\mathbb{C}^{n}$. 
In [20], Tamrazov obtained generalisations of Theorem 7 to finely holomorphic and finely hypoharmonic functions in finely open sets in $\mathbb{C}$.

2.3. Sharpness of results when subharmonicity can be used. Comparing (2.1) and (2.15) as conclusions (rather than assumptions) to (1.7), we see that the constant $C$ in (1.7) is equal to its best theoretically possible value 1 in (2.1) and (2.15). The same applies to other similar inequalities in the theorems in this Section.

2.4. Previous results for general majorants. Suppose that $\mu$ is a majorant, hence satisfying (1.1), but not necessarily bilogarithmically concave. Consider the problem of deducing (1.3) from (1.2). In [16], Theorem 3.1, p. 149 and Theorem 9.1, p. 166, Tamrazov obtained such results where $G$ can be fairly arbitrary as long as $\partial G$ is uniformly thick enough and $f$ is bounded, with $C$ in (1.3) depending on a quantity that measures the uniform lower density of the logarithmic capacity of the complement of $G$. Thus an absolute constant $C=108$ can be obtained whenever $G$ is a simply connected domain; the author improved this to $C=74$ in [9], Theorem A, p. 309 by essentially the same method. These proofs only use the fact that $\log |f|$ is subharmonic and do not make use of the argument of $f$. In contrast, while the proofs of Theorems 3-7 cited above are principally based on potential theory, some parts of the proofs use more strongly the assumption that $f$ is analytic by harnessing the fact that $\log |f|$ possesses a local harmonic conjugate function, namely, $\arg f$.

In [1], Aliev and Tamrazov obtained more refined bounds of the same kind, with $C$ depending on the same quantity as in [16] measuring the thickness of $\mathbb{C} \backslash G$. The new feature in their bounds for $\left|f\left(z_{1}\right)-f\left(z_{2}\right)\right|$ for $z_{2} \in G$, for a fixed $z_{1} \in \partial G$, is that they contain extra factors depending on the values of $g_{D}\left(z_{2}, \zeta_{j}\right)$ where the points $\zeta_{j}$ lie in the same component $D$ of $G$ as $z_{2}$ and satisfy $f\left(\zeta_{j}\right)=f\left(z_{1}\right)$. Here $g_{D}(z, w)$ is the Green's function of the domain $D$ with pole at $w \in D$.

The majorisation problem for a general majorant can be solved provided that an upper bound can be found in the special case when $\mu(t)=A \max \left\{t_{0}, t\right\}$ for some $A, t_{0}>0$, see Lemma 1.8 in [13]. In this case $\log \mu\left(e^{t}\right)=\log A+\max \left\{t, \log t_{0}\right\}$, which is a building block of general convex non-decreasing functions and hence about as far from concave as possible. For such majorants, it does not seem possible to get results by potential theory alone with an absolute constant $C$ on the right hand side of (1.3), independent of the thickness of $\partial G$. To better see the difficulty for general majorants, let us recall a discussion that we recently gave in [11]. The simplest multiply connected domain is the annulus $G=\{z \in \mathbb{C}: 1 / R<|z|<R\}$, where we choose $R>1$ 
to be large. If we take $z_{1} \in \partial G$ to be on the inner component of $\partial G$, we may assume, for all practical purposes (when $R$ is very large), that $z_{1}=0$ and $f(0)=0$. If we now take $\mu(t)=\max \{1, t\}$, our assumption (1.2) can be taken to be $|f(z)| \leq \max \{1,|z|\}$ for all $z \in \partial G$. As usual, write $M(r, f)=\max \{|f(z)|:|z|=r\}$. Then $M(1 / R, f) \leq 1$ and $M(R, f) \leq R$. If we choose $z_{2}=1 \in G$ in (1.3), we see that we would like to show that $|f(1)| \leq C$ for an absolute constant $C$. Hadamard's three-circles theorem states that $\log M(r, f)$ is a convex function of $\log r$, which implies that

$$
\log |f(1)| \leq \log M(1, f) \leq \frac{1}{2}(\log M(1 / R, f)+\log M(R, f)) \leq \frac{1}{2} \log R,
$$

which does not remain bounded above by an absolute constant. This indicates the problem that arises. Note that here the inner boundary component of $\partial G$ is "thin" when viewed from $z_{2}=1 \in G$.

This emphasises the fact that potential theoretic methods, based on using properties of $|f|$ alone via the subharmonic function $\log |f|$, cannot yield the best result in all cases. Thus it has been necessary to find an effective way of using the argument of $f$, which has the special property that if $G$ is bounded by finitely many disjoint Jordan curves, as we may assume by approximation (compare [9]), then $\arg f(z)$ changes by an integral multiple of $2 \pi$ when $z$ traverses any component of $\partial G$. In [13], we used approximation of $f$ by rational functions on the basis of approximation theorems proved in [9], to get first to a situation where it is easier to handle geometric properties of the function, and then considered the level curves of the resulting rational function, which amounts to taking into account the argument of that function.

\section{Preliminary Results}

3.1. Earlier results on majorisation. In order to avoid the repetition of certain arguments, we first note, as we did in [12], that the proofs given in [13] do, in fact, yield the following result.

Theorem 8. Suppose that $G$ is a non-empty open set in $\mathbb{C}$ with only bounded components and that $f$ is analytic in $G$ and continuous in $\bar{G}$, and let $\mu$ be a majorant of the form

$$
\mu(t)=A \max \left\{t_{0}, t\right\}
$$

for some positive constants $A$ and $t_{0}$. Suppose that $z_{1} \notin G$. If

$$
|f(z)| \leq \mu\left(\left|z-z_{1}\right|\right)
$$

for all $z \in \partial G$, then

$$
|f(z)| \leq C \mu\left(\left|z-z_{1}\right|\right)
$$


for all $z \in \bar{G}$ such that $\left|z-z_{1}\right|=t_{0}$, with $C=1728$.

In [12], Theorem 2.1 we inadvertently omitted to mention the condition $\left|z-z_{1}\right|=t_{0}$ in the conclusion of Theorem 8 but we did not use Theorem 8 in any other case in [12].

We remark, as we did in [12], that to understand why Theorem 8 is valid, one should see Lemma 1.8 in [13], and note that it is mentioned in the proof of [13], Theorem 1.11 that for majorants of the form (3.1), we obtain the desired conclusion with $C=1728$. In [13], we always took $A=1$ in (3.1), but this is immaterial since both $f$ and $\mu$ could be multiplied by the same positive constant.

The proof of [13], Theorem 1.11 is also based on the approximation result [9], Theorem 2, p. 309. Thus one needs to check that that result can be used to obtain Theorem 8 , but this is a matter of routine.

The following lemma was proved in [8], Lemma 2, pp. 249-250. It is an extension of a result of Tamrazov ([16], Lemma 4.1, p. 156) who had assumed that $\partial G$ is bounded and that $f$ is bounded.

Lemma 9. Let $G$ be an open set in $\mathbb{C}$ with at least one finite boundary point. Let $f$ be analytic in $G$ and continuous in $\bar{G}$, and suppose that

$$
f(z)=o\left(|z|^{2}\right)
$$

as $z \rightarrow \infty$ in each unbounded component of $G$. Then, for every positive $t$, we have

$$
\begin{gathered}
\sup \left\{\left|f\left(z_{1}\right)-f\left(z_{2}\right)\right|:\left|z_{1}-z_{2}\right| \leq t, z_{1}, z_{2} \in \bar{G}\right\} \\
=\sup \left\{\left|f\left(z_{1}\right)-f\left(z_{2}\right)\right|:\left|z_{1}-z_{2}\right| \leq t, z_{1} \in \partial G, z_{2} \in \bar{G}\right\} .
\end{gathered}
$$

3.2. Subharmonic functions. If $u$ is a subharmonic function in the open set $D$ in the plane and $r>0$, we write

$$
\begin{gathered}
B(r, u, D)=\sup \{u(z):|z|=r, z \in D\}, \\
I(r, u, D)=\frac{1}{2 \pi} \int_{r e^{i \theta} \in D} u\left(r e^{i \theta}\right) d \theta .
\end{gathered}
$$

When $D=\mathbb{C}$, we use the abbreviated notations

$$
B(r, u, \mathbb{C})=B(r, u), \quad I(r, u, \mathbb{C})=I(r, u) .
$$

The proofs of Theorems 1 and 2 both use to a substantial extent the ideas in [4]. Since certain details differ, we will not be able to just apply the theorems in [4]. Nonetheless, in order to put the differences and the similarities in perspective, let us quote [4], Theorem 2, p. 244, so that the reader will be able to compare certain statements obtained in the course of our proofs to the results proved in [4]. 
Theorem 10. Suppose that $u$ is a positive subharmonic function in an open set $G$ in the plane, whose complement contains at least one finite point, and that

$$
\limsup _{z \rightarrow \zeta, z \in G} u(z) \leq 0
$$

as $z$ approaches any finite boundary point $\zeta$ of $G$ from inside $G$. Then there exists $\beta \in(0,+\infty]$ such that

$$
\lim _{r \rightarrow \infty} \frac{B(r, u, G)}{\log r}=\lim _{r \rightarrow \infty} \frac{I(r, u, G)}{\log r}=\beta .
$$

Suppose further that $\beta<+\infty$, and that $u$ is harmonic in $G$ and possesses there a local conjugate $v$, such that for some $\alpha$, where $0<\alpha \leq 1$, and some positive $R_{0}$,

$$
F(z)=z^{1-\alpha} \exp (u+i v)
$$

remains single-valued in $G \cap\left\{z \in \mathbb{C}:|z|>R_{0}\right\}$. Then $G$ contains the set $\{z \in \mathbb{C}:|z|>R\}$ for some $R>R_{0}$, the function $F$ has a pole of order $p$, say, at infinity, and $\beta=\alpha+p-1$.

Thus in the situation of Theorem 10, the point at infinity is an isolated boundary point of $G$ in the Riemann sphere $\overline{\mathbb{C}}=\mathbb{C} \cup\{\infty\}$.

3.3. Growth of majorants. Finally, we note that for a majorant $\mu$ it follows from (1.1) that $\mu\left(2^{n} t\right) \leq 2^{n} \mu(t)$ for all $t \geq 0$ and all positive integers $n$. Hence if $\lambda>1$ and if the positive integer $n$ is chosen so that $2^{n-1}<\lambda \leq 2^{n}$, we obtain

$$
\mu(\lambda t) \leq \mu\left(2^{n} t\right) \leq 2^{n} \mu(t) \leq 2 \lambda \mu(t) .
$$

\section{Proof of Theorem 1}

We will present the proof of Theorem 2 in Section 5. For the time being, we assume that Theorem 2 is valid.

Let the assumptions of Theorem 1 be satisfied. Thus $\mu$ is a majorant, $G$ is an open set in the plane with at least two finite boundary points, $f$ is analytic in $G$ and continuous in $\bar{G}, z_{1}$ is a given boundary point of $G$, and we assume that (1.5) holds for all $z_{2} \in \partial G$.

We note that by (1.1), we either have $\mu(t)=0$ for all $t \geq 0$ or $\mu(t)>0$ for all $t>0$ (compare [12]). We will deal with the case $\mu \equiv 0$ later. For the time being, we therefore assume that $\mu(t)>0$ for all $t>0$.

We generally follow the proof of Theorems 1, 2, and 3 in [4].

Pick $z_{2} \in G$ and let $U$ be the component of $G$ containing $z_{2}$. If $U$ is bounded, then it follows from Theorem 8, applied to $U$ instead of $G$ 
and to $f(z)-f\left(z_{1}\right)$ instead of $f$, and noting that $z_{1} \notin U$, that (1.3) holds with $C=3456$. Namely, if $t_{0}=\left|z_{1}-z_{2}\right|$ and

$$
\mu_{1}(t)=2 \mu\left(t_{0}\right) t_{0}^{-1} \max \left\{t, t_{0}\right\}
$$

then $\left|f(z)-f\left(z_{1}\right)\right| \leq \mu_{1}\left(\left|z-z_{1}\right|\right)$ for all $z \in \partial U$, so that by Theorem 8 , we have

$$
\left|f\left(z_{2}\right)-f\left(z_{1}\right)\right| \leq 1728 \mu_{1}\left(\left|z_{2}-z_{1}\right|\right)=3456 \mu\left(\left|z_{1}-z_{2}\right|\right) .
$$

Thus we may assume that $U$ is unbounded.

Set $\rho=\left|z_{1}-z_{2}\right| / 2$ and define

$$
V=U \cap\left\{z \in \mathbb{C}:\left|z-z_{1}\right|>\rho\right\} .
$$

The unbounded open set $V$ need not be connected. Then $\partial V \subset\{z \in$ $\left.\mathbb{C}:\left|z-z_{1}\right|=\rho\right\} \cup \partial G$. Since $f$ is continuous in $\bar{G}$, there is a positive number $M_{0}$ with $M_{0} \geq 1$ such that

$$
\left|f(z)-f\left(z_{1}\right)\right| \leq M_{0} \mu\left(\left|z_{1}-z_{2}\right|\right)
$$

whenever $z \in\left\{\zeta \in \mathbb{C}:\left|\zeta-z_{1}\right|=\rho\right\} \cap \bar{G}$.

By (1.5) and (3.8), it follows for $z \in \partial G$ that

$$
\left|f(z)-f\left(z_{1}\right)\right| \leq \mu\left(\left|z_{1}-z_{2}\right|\right) \quad \text { if }\left|z-z_{1}\right| \leq\left|z_{1}-z_{2}\right|
$$

and

$$
\left|f(z)-f\left(z_{1}\right)\right| \leq \frac{2\left|z-z_{1}\right| \mu\left(\left|z_{1}-z_{2}\right|\right)}{\left|z_{1}-z_{2}\right|} \quad \text { if }\left|z-z_{1}\right|>\left|z_{1}-z_{2}\right| .
$$

Define, for $z \in \bar{G} \backslash\left\{z_{1}\right\}$,

$$
u_{1}(z)=\log \left|f(z)-f\left(z_{1}\right)\right|-\log \left(M_{0} \mu\left(\left|z_{1}-z_{2}\right|\right) \frac{2\left|z-z_{1}\right|}{\left|z_{1}-z_{2}\right|}\right) .
$$

Then $u_{1}$ is defined and upper semicontinuous in $\bar{G} \backslash\left\{z_{1}\right\}$ as a function with values in $\mathbb{R} \cup\{-\infty\}$, and is subharmonic in $V$. Our first aim is to show that $u_{1} \leq 0$ in $V$ unless $G$ contains a neighbourhood of infinity. This will not yield anything like (1.7) since we have no information on $M_{0}$, but it will give us a starting point.

Suppose that $z \in \partial V$. If $z \in \partial G$ and $\left|z-z_{1}\right|>\left|z_{1}-z_{2}\right|$, then

$$
u_{1}(z) \leq-\log M_{0} \leq 0
$$

since $M_{0} \geq 1$. If $z \in \partial G$ and $\left|z-z_{1}\right| \leq\left|z_{1}-z_{2}\right|$, then, since $z \in \partial V$, we have $\left|z-z_{1}\right| \geq \rho=\left|z_{1}-z_{2}\right| / 2$ so that

$$
u_{1}(z) \leq-\log \left(\frac{2\left|z-z_{1}\right|}{\left|z_{1}-z_{2}\right|}\right)-\log M_{0} \leq 0 .
$$

If $\left|z-z_{1}\right|=\rho$ and $z \in G$ then $\left|f(z)-f\left(z_{1}\right)\right| \leq M_{0} \mu\left(\left|z_{1}-z_{2}\right|\right)$ so that $u_{1}(z) \leq 0$. 
We conclude that $u_{1}(z) \leq 0$ for all $z \in \partial V$.

If $u_{1}$ is bounded above in $V$, it now follows from the maximum principle for subharmonic functions (see [6], Theorem 5.16, p. 232) applied to each component of $V$ separately that $u_{1}(z) \leq 0$ for all $z \in V$.

Next suppose that $u_{1}$ is not bounded above in $V$. Let $W$ be a component of the set $\left\{z \in V: u_{1}(z)>0\right\}$. Then $W$ is necessarily unbounded. For if $W$ is bounded, we note that $u_{1}$ is clearly bounded above in every compact subset of $\bar{G}$ not containing $z_{1}$, and consequently $u_{1}$ is bounded above in $W$. Since $u_{1} \leq 0$ on $\partial W$, it again follows that $u_{1} \leq 0$ in $W$, a contradiction. Thus $W$ is unbounded.

Since $u_{1} \leq 0$ on $\partial V$, it follows that $u_{1}(z)=0$ for all $z \in \partial W$. We define the function $u$ in $\mathbb{C}$ by setting $u(z)=u_{1}(z)$ for all $z \in W$ and $u(z)=0$ for all $z \in \mathbb{C} \backslash W$. Then $u$ is a non-negative non-constant subharmonic function in the plane.

We would like to apply [4], Theorem 2, p. 244 (Theorem 10 above) to $u$, taking $\alpha=1$. We prefer to recall the details of the proof to some extent. This has the additional advantage of explaining why results of this nature can be true at all, guaranteeing that under suitable circumstances an unbounded domain has to contain a neighbourhood of infinity. This conclusion is one of the essential elements of the statement of Theorem 1.

Standard convexity theorems (see [6], Theorem 2.14, p. 67, and [4], p. 245) show that there is $\beta \in(0, \infty]$ such that the following limits exist and are both equal to $\beta$ :

$$
\lim _{r \rightarrow \infty} \frac{B(r, u)}{\log r}=\lim _{r \rightarrow \infty} \frac{I(r, u)}{\log r}=\beta .
$$

Since $|f(z)|=O\left(|z|^{q}\right)$ for some finite $q$ as $z \rightarrow \infty$ in $U$, it follows that $\beta$ is finite. Since $u$ is of order zero (for the definition, see [6], Definitions 4.1 and 4.2 , p. 143) and is finite at the origin, it has the representation (see [6], p. 155)

$$
u(z)=u(0)+\int_{\mathbb{C}} \log \left|1-\frac{z}{\zeta}\right| d \nu(\zeta)
$$

where $\nu$ denotes the Riesz measure of $u$. Let $n(r)$ denote the total measure of $\nu$ in $\{z \in \mathbb{C}:|z|<r\}$. Then by Nevanlinna's first fundamental theorem for subharmonic functions ([6], (3.9.6), p. 127) we have

$$
I(r, u)=\int_{0}^{r} n(t) \frac{d t}{t}+u(0) .
$$

This implies that

$$
\beta=\lim _{r \rightarrow \infty} n(r)
$$


Thus the total Riesz measure of the plane is finite and is equal to $\beta$.

Since $u$ has order zero, it follows from the extension of Wiman's theorem due to Heins [7] that the function

$$
A(r)=\inf \{u(z):|z|=r\}
$$

is unbounded as $r \rightarrow \infty$. Since $A(r)=0$ for each $r$ such that the circle $\{z \in \mathbb{C}:|z|=r\}$ intersects the complement of $W$, and in particular if this circle intersects the complement of $G$, it follows that $W$, and hence $G$, contains a sequence of circles $\left\{z \in \mathbb{C}:|z|=r_{n}\right\}$ where $r_{n}$ increases to infinity. Thus $G$ has only one unbounded component.

Note that $u(z)$ has a local conjugate

$$
v(z)=\arg \left(f(z)-f\left(z_{1}\right)\right)-\arg \left(z-z_{1}\right)
$$

in $W$ such that

$$
F(z)=\exp (u+i v)=C \frac{f(z)-f\left(z_{1}\right)}{z-z_{1}}
$$

is single-valued in $W$, where $C=\left|z_{1}-z_{2}\right| /\left(2 M_{0} \mu\left(\left|z_{1}-z_{2}\right|\right)\right)$ is a constant.

By (4.5) applied to $r=r_{n}$ we obtain, applying also the CauchyRiemann equations to $u$ and $v$,

$$
\begin{aligned}
n(r) & =r \frac{d}{d r} I(r, u)=\frac{1}{2 \pi} \int_{0}^{2 \pi} r \frac{\partial}{\partial r} u\left(r e^{i \theta}\right) d \theta \\
& =\frac{1}{2 \pi} \int_{0}^{2 \pi} \frac{\partial}{\partial \theta} v\left(r e^{i \theta}\right) d \theta=p_{n},
\end{aligned}
$$

where $p_{n}$ is an integer, since $F$ given by (4.7) is single-valued.

Since $n(r)$ is increasing and bounded, it follows that $p_{n}$ is constant for all large $n$ and hence $n(r)$ is constant and is equal to $\beta$ (which therefore is a positive integer) for all large $r$, say for $r \geq R_{1}$. Thus there is no Riesz measure of $u$ in the set $\left\{z \in \mathbb{C}:|z|>R_{1}\right\}$ and so $u$ is harmonic there. Since $u \geq 0$ so that the value 0 is the minimum value of $u$, since a non-constant harmonic function will not attain its minimum at an interior point of a domain where it is harmonic, and since $u=0$ outside $W$, it follows that $W$, and hence $G$, contains a neighbourhood of infinity. Hence $F$ has an isolated singularity at infinity, and due to the growth condition on $f$, the singularity is either removable or a pole. Since $|F|$ behaves like $|f| /|z|$ when $z$ is large, and $|F(z)|$ grows like $|z|^{\beta}$ where $\beta \geq 1$, we see that $f$ has a pole of order at least two at infinity.

We conclude that if it is not the case that $u_{1} \leq 0$ in $V$, then $G$ contains a neighbourhood of infinity and $f$ has a pole of order at least two at infinity. This means that we are then in case (ii) of Theorem 1. 
Let us divide the rest of the argument into two cases: when $\partial G$ is bounded and when $\partial G$ is unbounded.

Suppose that $\partial G$ is bounded. Then $G$ contains exactly one unbounded component, which, in our argument, must therefore be $U$, and $U$ contains a neighbourhood of infinity.

If $f$ has a pole of order at least two at infinity, then (1.7) cannot hold for all $z \in G$ since it follows from (1.1) that $\mu(t)=O(t)$ as $t \rightarrow \infty$.

If $f$ has no pole at infinity, then $f$ is bounded in $\bar{G}$, in which case it follows from Theorem 1.2 in [12] that (1.7) holds for all $z_{2} \in G$ with $C=3456$.

Suppose that $f$ has a simple pole at infinity. Since $\mu(t)$ may grow at the same rate as $t$ as $t \rightarrow \infty$, there remains the possibility that (1.7) might hold for all $z_{2} \in G$ with $C=3456$. However, the assumption that (1.5) holds for all $z_{2} \in \partial G$, and even the stronger assumption that (1.5) holds for all $z_{1}, z_{2} \in \partial G$, can give us information about $\mu(t)$ only for $0 \leq t \leq \operatorname{diam} G$. Since $\mu$ could now be a bounded function, we are not able to deduce that (1.7) will necessarily hold for all $z_{2} \in G$ for any particular constant $C$. This is the best that we can say when $\partial G$ is bounded, at least in the absence of further assumptions, in the case when $f$ has a simple pole at infinity.

Suppose that $\partial G$ is unbounded. Then also $\partial V$ is unbounded (for otherwise, $V$, and hence $G$, contains a neighbourhood of infinity). From what we did above, it follows that in this case $u_{1}$ is bounded above in $V$, and furthermore, $u_{1}(z) \leq 0$ for all $z \in \bar{V}$. This implies that

$$
\left|f(z)-f\left(z_{1}\right)\right| \leq \frac{2 M_{0} \mu\left(\left|z_{1}-z_{2}\right|\right)}{\left|z_{1}-z_{2}\right|}\left|z-z_{1}\right|
$$

for all $z \in V$, so that $|f(z)|=O(|z|)$ as $z \rightarrow \infty$ in $V$.

Define

$$
\mu_{1}(t)=\frac{2 \mu\left(\left|z_{1}-z_{2}\right|\right)}{\left|z_{1}-z_{2}\right|} \max \left\{t,\left|z_{1}-z_{2}\right|\right\}
$$

for $t \geq 0$. Then $\mu_{1}$ is a majorant, and by (3.8), we have $\mu(t) \leq \mu_{1}(t)$ for all $t \geq 0$. Hence by (1.5), we have $\left|f(z)-f\left(z_{1}\right)\right| \leq \mu_{1}\left(\left|z-z_{1}\right|\right)$ for all $z \in \partial G$ and in particular whenever $z \in \partial V$ and $\left|z-z_{1}\right|>\rho$. It follows that

$$
\limsup _{z \rightarrow \infty, z \in \partial V} \frac{|f(z)|}{|z|} \leq \frac{2 \mu\left(\left|z_{1}-z_{2}\right|\right)}{\left|z_{1}-z_{2}\right|} .
$$

We apply Theorem 2 to $f$ in $V$ (instead of $G$ ) and conclude that

$$
\limsup _{z \rightarrow \infty, z \in \bar{V}} \frac{|f(z)|}{|z|} \leq \frac{2 \mu\left(\left|z_{1}-z_{2}\right|\right)}{\left|z_{1}-z_{2}\right|} .
$$


Since $V$ need not be connected even if $U$ is connected, this is one reason why we need to have Theorem 2 formulated for an open set rather than for just a domain. If we tried to replace $V$ by a component of $V$ in this part of the argument, we would run into other problems in the proof of Theorem 1.

Pick $\varepsilon>0$. By (4.8), we can find $R_{2}$ with $R_{2}>\left|z_{1}-z_{2}\right|$ such that

$$
\left|f(z)-f\left(z_{1}\right)\right| \leq\left|z-z_{1}\right|\left(\frac{2 \mu\left(\left|z_{1}-z_{2}\right|\right)}{\left|z_{1}-z_{2}\right|}+\varepsilon\right)
$$

for all $z \in \bar{V}$ with $\left|z-z_{1}\right| \geq R_{2}$.

Now define

$$
\mu_{2}(t)=\left(\frac{2 \mu\left(\left|z_{1}-z_{2}\right|\right)}{\left|z_{1}-z_{2}\right|}+\varepsilon\right) \max \left\{t,\left|z_{1}-z_{2}\right|\right\}
$$

for $t \geq 0$. Then $\mu_{2}$ is a majorant, and $\mu(t) \leq \mu_{1}(t) \leq \mu_{2}(t)$ for all $t \geq 0$.

Let $V_{1}$ be the component of $U \cap\left\{z \in \mathbb{C}:\left|z-z_{1}\right|<R_{2}\right\}$ containing $z_{2}$. If $z \in \partial V_{1}$ then $z \in \partial G$ or $z \in\left\{\zeta:\left|\zeta-z_{1}\right|=R_{2}\right\} \cap U$. In both cases we have $\left|f(z)-f\left(z_{1}\right)\right| \leq \mu_{2}\left(\left|z-z_{1}\right|\right)$. Since $\mu_{2}$ is of the special form indicated by (3.1), it follows from Theorem 8 , applied to $f$ in $V_{1}$, that

$$
\left|f\left(z_{2}\right)-f\left(z_{1}\right)\right| \leq 1728 \mu_{2}\left(\left|z_{1}-z_{2}\right|\right) .
$$

Since $\varepsilon>0$ is arbitrary, we conclude that (1.7) holds for our point $z_{2}$ with $C=3456$.

This completes the proof of Theorem 1 for a fixed $z_{1} \in \partial G$, subject to Theorem 2.

Suppose then that (1.5) holds for all $z_{1}, z_{2} \in \partial G$. We have already proved that if (ii) of Theorem 1 does not hold, then (1.3) holds with $C=3456$ whenever $z_{1} \in \partial G$ and $z_{2} \in G$. This implies, in particular, that $f(z)=O(|z|)$ as $z \rightarrow \infty$ in any unbounded component of $G$, since $\mu(t)=O(t)$ as $t \rightarrow \infty$. Now pick any $w_{1}, w_{2} \in \bar{G}$ and set $h=w_{2}-w_{1}$ and $t=|h|>0$. Thus we may apply Lemma 9 and conclude that there are $z_{1} \in \partial G$ and $z_{2} \in G$ with $\left|z_{1}-z_{2}\right| \leq t$ such that

$$
\left|f\left(w_{1}\right)-f\left(w_{2}\right)\right| \leq\left|f\left(z_{1}\right)-f\left(z_{2}\right)\right| \leq C \mu\left(\left|z_{1}-z_{2}\right|\right) \leq C \mu\left(\left|w_{1}-w_{2}\right|\right),
$$

where $C=3456$. We have now dealt with the case when the majorant $\mu$ is not identically zero.

Suppose then that $\mu(t) \equiv 0$. We may assume that we are not in the case (ii) of Theorem 1. We proceed in the same way as in [12]. Set $g(z)=f(z)-f\left(z_{1}\right)$. Thus our assumption is that $g \equiv 0$ on $\partial G$. We wish to conclude that $g \equiv 0$ in $G$. This is clear by the maximum modulus principle if each component of $G$ is bounded, so let us assume 
that $G$ has at least one unbounded component. Note that (1.6) holds with $f$ replaced by $g$.

We now apply the result of Fuchs [3]. Pick any real number $\varepsilon>0$. Since $|g(z)| \leq \varepsilon$ for all $z \in \partial G$ and since (1.6) holds with $f$ replaced by $g$, [3], Theorem 1, p. 285, applied to $g / \varepsilon$, not in $G$ but separately in each unbounded component of $G$, implies that $|g(z)| \leq \varepsilon$ for all $z$ in each unbounded component of $G$, and hence for all $z \in G$. Since $\varepsilon>0$ can be arbitrarily small in this conclusion, we see that $|g(z)|=0$ and hence $g(z)=0$ for all $z \in G$.

This completes the proof of Theorem 1, subject to Theorem 2 .

Remark. Consider the last situation where $g \equiv 0$ on $\partial G$. If the boundary of an unbounded component $D$ of $G$ contains a continuum, one can try to consider a bounded simply connected subdomain $V$ of $D$ such that $\partial V$ contains a continuum on which $g$ vanishes identically; if $\partial D$ is not connected and is topologically complicated, it is perhaps not clear that such a domain $V$ can be found. If $V$ exists, then, since $g$ is bounded in $V$, it follows, after a conformal mapping taking the unit disk onto $V$, from theorems for bounded analytic functions in the unit disk (for example, [2], Theorem 2.2, p. 17) that $g$ vanishes identically in $V$. By analytic continuation, $g$ vanishes identically in $D$.

If a simply connected domain $V$ as above cannot be found, choose $V$ as above but of any connectivity and let $\varphi$ be a universal covering mapping of the unit disk $\mathbb{D}$ onto $V$. Then $g \circ \varphi$ is bounded in $\mathbb{D}$, and by Privalov's theorem ([14], Theorem 10.14, p. 325), we obtain $g \circ \varphi \equiv 0$ in $\mathbb{D}$, hence $g \equiv 0$ in $V$ and hence in $D$.

This leaves only the case when $G$ is connected and is equal to a domain obtained by removing from the whole plane a closed set containing no continuum, and $g$ grows at most like a polynomial as $z \rightarrow \infty$ in $G$. If (ii) of Theorem 1 does not hold, then $\partial G$ is unbounded and $g$ has infinitely many zeros if we include all the points on $\partial G$ among the zeros of $g$ in this loose sense. Any isolated boundary point of the domain is removable for $g$ so that we replace $G$ by a larger domain, if necessary, and assume that no boundary point of $G$ is isolated. If the boundary of the domain so enlarged is bounded, possibly empty, we are again in the situation (ii) of Theorem 1, and then $g$ is analytic in a neighbourhood of infinity and has at most a pole at infinity. Therefore $g$ has only finitely many zeros in a small neighbourhood of infinity, a contradiction unless $g \equiv 0$ in $G$.

Thus we may assume that for the enlarged domain, still denoted by $G$, no boundary point is isolated, and $\partial G$ is unbounded. The boundary of $G$ then resembles a Cantor set (indeed, if we add the point at 
infinity to $\partial G$ we get a Cantor subset of the Riemann sphere), $g$ vanishes on $\partial G$ and grows at most like a power at infinity. It might be of interest to know whether a result more elementary than or historically earlier than that of Fuchs [3] could be used to handle this case and prove that then $g$ vanishes identically in $G$. Considering a universal covering mapping $\varphi$ of $\mathbb{D}$ onto $G$ and using Privalov's theorem on $g \circ \varphi$ ought to work since $\varphi^{-1}(\infty)$ should be a small subset of $\partial \mathbb{D}$.

We could again note that for any $\varepsilon>0$, the function $u(z)=\log |g(z) / \varepsilon|$ is subharmonic in the plane so that so is $u^{+}=\max \{0, u\}$, and by applying to $u^{+}$the method of proof of [4], Theorem 2, which we have also used above in the proof of Theorem 1, we could conclude that $G$ contains a neighbourhood of infinity, a contradiction. However, [4] was written after the work of Fuchs [3].

\section{Proof of Theorem 2}

Let the assumptions of Theorem 2 be satisfied. If

$$
\limsup _{z \rightarrow \infty, z \in \partial G}|f(z) / z|=\infty
$$

then the assertion of Theorem 2 is trivially true. Thus we may and will assume that

$$
\limsup _{z \rightarrow \infty, z \in \partial G}|f(z) / z|=A_{1}<\infty .
$$

Here $A_{1} \geq 0$. Choose any $A>A_{1}$. Thus $A>0$. Our assumption implies that there is a positive number $R$ such that

$$
|f(z)| \leq A|z| \quad \text { whenever } z \in \partial G \text { and }|z| \geq R .
$$

We will prove that

$$
\limsup _{z \rightarrow \infty, z \in \bar{G}} \frac{|f(z)|}{|z|} \leq A .
$$

Since $A$ is an arbitrary number greater than $A_{1}$, this then implies (1.9), and, as noted after the statement of Theorem 2, (1.9) implies (1.8).

5.1. Boundedness of $|f(z) / z|$. Our first task is to prove that

$$
\limsup _{z \rightarrow \infty, z \in G} \frac{|f(z)|}{|z|}<\infty .
$$

Choose any $R_{0}>0$. The assumptions of Theorem 2 imply that there is $M>0$ such that

$$
|f(z)|<M|z|
$$

whenever $z \in \bar{G}$ and $|z|=R_{0}$, and whenever $z \in \partial G$ and $|z| \geq R_{0}$. Write $G_{1}=G \cap\left\{z \in \mathbb{C}:|z|>R_{0}\right\}$. Define $\varphi(z)=f(z) /(M z)$ so 
that $\varphi$ is continuous in $\overline{G_{1}}$ and analytic in $G_{1}$, and $|\varphi(z)| \leq 1$ for all $z \in \partial G_{1}$.

We will prove that $|\varphi(z)| \leq 1$ for all $z \in G_{1}$, which clearly implies (5.3). To get a contradiction, suppose that this is not the case. Define

$$
G_{2}=\left\{z \in G_{1}:|\varphi(z)|>1\right\} \neq \emptyset,
$$

let $G_{3}$ be an arbitrary connected component of $G_{2}$, and define the nonnegative non-constant function $u(z)$ in the plane by setting $u(z)=$ $\log |\varphi(z)|$ when $z \in G_{3}$, and $u(z)=0$ when $z \notin G_{3}$. Then $u$ is subharmonic in the plane. Note that $G_{3}$ must be contained in a component of $G_{1}$, and hence in a component of $G$. If $G_{3}$ is bounded, then the fact that $u=0$ on $\partial G_{3}$ implies by the maximum principle that $u \leq 0$ in $G_{3}$, which is a contradiction. Hence $G_{3}$ is unbounded, and is contained in an unbounded component of $G$. Thus by (1.6), there is a positive real number $q$ such that

$$
u(z) \leq(q-1) \log |z|+O(1)
$$

as $z \rightarrow \infty$ in $G_{3}$. It follows that (5.4) holds as $z \rightarrow \infty$ in $\mathbb{C}$. As in the proof of Theorem 1 (or applying Theorem 10 to $u$ in $G_{3}$ with $\alpha=1$ ) we find that $G_{3}$ contains a neighbourhood of infinity. Thus $G$ contains a neighbourhood of infinity, which contradicts our assumption that $\partial G$ is unbounded. This contradiction proves (5.3).

5.2. Estimating the upper limit of $|f(z) / z|$. Pick $w \in \mathbb{C} \backslash G$. Define

$$
F(z)=\frac{f(z)}{A(z-w)} .
$$

Then $F$ is analytic in $G$ and continuous in $\bar{G} \backslash\{w\}$. Furthermore, $F$ is bounded in $\bar{G}$ outside a small neighbourhood of $w$.

Pick $\varepsilon>0$. We may choose a positive number $R_{1}$ depending on $w, A, R, \varepsilon$ and with $R_{1}>|w|+1$ such that

$$
|F(z)| \leq 1+\varepsilon \quad \text { whenever } z \in \partial G \quad \text { and }|z| \geq R_{1} \text {. }
$$

Since $\varepsilon>0$ is arbitrary, in order to prove (5.2) it suffices to prove that

$$
\limsup _{z \rightarrow \infty, G \in G}|F(z)| \leq 1+2 \varepsilon .
$$

Define $V=G \cap\left\{z \in \mathbb{C}:|z|>R_{1}\right\}$ and

$$
W=\{z \in V:|F(z)|>1+2 \varepsilon\} .
$$

Then $W$ is an open set, possibly empty. If $W$ is not empty, then $W$ need not be connected. 
If $W$ is bounded, then (5.6) holds. Suppose that $W$ is unbounded. Define $D=\left\{z \in \mathbb{C}:|z|>R_{1}\right\}$. For $z \in D$, we define the function $u$ by

$$
u(z)=\log |F(z)|-\log (1+2 \varepsilon)>0
$$

if $z \in W$, and $u(z)=0$ if $z \in D \backslash W$. By (5.5), we have $\lim _{z \rightarrow \zeta} u(z)=0$ as $z$ approaches any boundary point $\zeta$ of $W$ from within $W$, and any such $\zeta$ must lie in $G$. Hence $u$ is subharmonic and bounded above in $D$.

To get a contradiction, suppose that (5.6) does not hold, which means that

$$
\limsup _{z \rightarrow \infty} u(z)>0
$$

Since $u \leq 0$ (indeed, $u=0$ ) on $E=D \backslash W$, this means that $E$ is a thin set (see [5], p. 388).

We denote the logarithmic capacity of a compact set $K$ by cap $K$ (for the definition, see [14], p. 332). Fix a real number $k$ with $k>1$. Choose a positive integer $m_{0}$ so that $k^{m_{0}-1}>R_{1}$. For positive integers $m \geq m_{0}$, let us write $E_{m}=E \cap\left\{z \in \mathbb{C}: k^{m-1} \leq|z| \leq k^{m}\right\}$ and

$$
C_{m}=\operatorname{cap} E_{m} \text {. }
$$

Recall that we define $\log ^{+} 0=0$ and for a positive real number $x$, we set $\log ^{+} x=\max \{0, \log x\}$. By the Wiener criterion (see [5], Theorem 7.5, p. 395), the set $E$ is thin if, and only if,

$$
\sum_{m=m_{0}}^{\infty} \frac{m}{1+\log ^{+}\left(1 / C_{m}\right)}<\infty .
$$

Since our set $E=D \backslash W$ is thin, it follows that for all sufficiently large $m$, we have

$$
\frac{m}{1+\log ^{+}\left(1 / C_{m}\right)}<1
$$

that is,

$$
C_{m}<e^{1-m} \text {. }
$$

For any compact set $K$, the definition of capacity as the transfinite diameter implies that cap $K \geq \operatorname{cap} K^{*}$ where $K^{*}=\{|z|: z \in K\}$. If $E=D \backslash W$ were to intersect the circle $S(0, r)=\{z \in \mathbb{C}:|z|=r\}$ for every $r \in\left[k^{m-1}, k^{m}\right]$, it would follow that (see [14], (20), p. 335)

$$
C_{m} \geq \operatorname{cap}\left[k^{m-1}, k^{m}\right]=(k-1) k^{m-1} / 4,
$$

which gives a contradiction with (5.7) when $m$ is sufficiently large. It follows that $W$, and hence $G$, contains circles with arbitrarily large radius. Hence the open set $G$ has only one unbounded component.

Since thin sets exist, we cannot get any further towards obtaining a contradiction by using only the fact that $u$ is subharmonic. We 
now have to make more careful use of the fact that $u$ arises from an analytic function $F$, and in particular, we need to use the properties of the argument of $F$. We follow the ideas of [4].

Define the function $u_{1}(z)=u(1 / z)$ for $0<|z|<1 / R_{1}$. Then $u_{1}$ is subharmonic and bounded above in $D_{1}^{\prime}=\left\{z \in \mathbb{C}: 0<|z|<1 / R_{1}\right\}$ and $b=\limsup _{z \rightarrow 0} u_{1}(z)$ is finite. Hence by [6], Theorem 5.18, p. 237, we obtain a subharmonic function, still denoted by $u_{1}$ in $D_{1}=\{z \in$ $\left.\mathbb{C}:|z|<1 / R_{1}\right\}$ by setting $u_{1}(0)=b$.

For $z \in D_{1}$, the function $u_{1}$ has the representation ([6], Theorem 3.9, p. 104)

$$
u_{1}(z)=u_{2}(z)+\int_{D_{1}} \log |z-\zeta| d \nu(\zeta)
$$

where $\nu$ denotes the Riesz measure of $u_{1}$ ([6], p. 113), and where $u_{2}$ is harmonic in $D_{1}$ and continuous in $\overline{D_{1}}$ (in view of the dependence of $u_{1}$ on $F)$. Let $n(r)$ denote the total measure of $\nu$ in $\{z \in \mathbb{C}:|z|<r\}$. Integrating (5.8) when $z$ traces a circle of radius $r \in\left(0,1 / R_{1}\right)$, using the fact that $\int_{0}^{2 \pi} \log \left|e^{i \theta}-\zeta\right| d \theta=2 \pi \log ^{+}|\zeta|$, and integrating by parts, we obtain

$$
\begin{aligned}
I\left(r, u_{1}, D_{1}\right) & =I\left(r, u_{2}, D_{1}\right)+n\left(1 / R_{1}\right) \log \left(1 / R_{1}\right)-\int_{r}^{1 / R_{1}} n(t) \frac{d t}{t} \\
& =u_{2}(0)+n\left(1 / R_{1}\right) \log \left(1 / R_{1}\right)-\int_{r}^{1 / R_{1}} n(t) \frac{d t}{t} .
\end{aligned}
$$

In each simply connected subdomain of $W_{1}=\{z \in \mathbb{C}: 1 / z \in W\} \subset$ $D_{1}^{\prime}$, we can write

$$
u_{1}(z)+i v(z)=\log \frac{f(1 / z)}{A(1+2 \varepsilon)(1 / z-w)}
$$

where $v$ is a local harmonic conjugate of $u_{1}$. Since the multi-valued nature of $v$ can only mean replacing $v$ by $v+C_{0}$ for a constant $C_{0}$ if we get to a different branch of $v$ by analytic continuation, the first order partial derivatives of $v$ are well defined in all of $W_{1}$.

If a circle $S(0, r)$ is contained in $W_{1}$, we obtain by (5.9), applying also the Cauchy-Riemann equations to $u_{1}$ and $v$, that

$$
\begin{aligned}
n(r) & =r \frac{d}{d r} I\left(r, u_{1}, D_{1}\right)=\frac{1}{2 \pi} \int_{0}^{2 \pi} r \frac{\partial}{\partial r} u_{1}\left(r e^{i \theta}\right) d \theta \\
& =\frac{1}{2 \pi} \int_{0}^{2 \pi} \frac{\partial}{\partial \theta} v\left(r e^{i \theta}\right) d \theta=p(r)
\end{aligned}
$$


where $p(r)$ is an integer, since the function

$$
f(1 / z) /(A(1+2 \varepsilon)(1 / z-w))
$$

is single-valued in $W_{1}$.

Assuming that $n(r)$ does not vanish identically, choose a sequence of radii $r_{n}$ less than $1 / R_{1}$ and decreasing to 0 such that each circle $S\left(0, r_{n}\right)$ is contained in $W_{1}$. Then $p\left(r_{n}\right)$ is a non-increasing sequence of non-negative integers, so that there are only finitely many distinct values among the $p\left(r_{n}\right)$, and so the sequence $p\left(r_{n}\right)$ is constant from some value of $n$ onwards. This means that there is no Riesz measure of $u_{1}$ in the punctured disk $D_{2}=\{z \in \mathbb{C}: 0<|z|<\rho\}$ for some $\rho \in\left(0,1 / R_{1}\right)$. Thus $u_{1}$ is harmonic in $D_{2}$, so that $u$ is harmonic in $D_{3}=\{z \in \mathbb{C}:|z|>1 / \rho\}$. Thus $u$ cannot attain its minimum value 0 in $D_{3}$ so that $u$ is positive there, and hence $D_{3} \subset W \subset G$. But this means that $\partial G$ is bounded, which is against the assumption of Theorem 2. This contradiction proves (5.6) and hence (5.2).

This completes the proof of Theorem 2 and hence also that of Theorem 1.

\section{REFERENCES}

[1] T. Aliyev and P. M. Tamrazov, Contour-solid theorem for meromorphic functions taking multivalence into account, pp. 469-475 in Progress in analysis, Vol. I, II (Berlin, 2001), World Sci. Publ., River Edge, NJ, 2003.

[2] P.L. Duren, Theory of $H^{p}$ spaces, Academic Press, New York-London, 1970.

[3] W. H. J. Fuchs, A Phragmén-Lindelöf theorem conjectured by D. J. Newman, Trans. Amer. Math. Soc. 267 (1981), 285-293.

[4] F.W. Gehring, W.K. Hayman, and A. Hinkkanen, Analytic functions satisfying Hölder conditions on the boundary, J. Approx. Theory 35 (1982), 243-249.

[5] W.K. Hayman, Subharmonic Functions, vol. 2, Academic Press, London, 1989.

[6] W.K. Hayman and P.B. Kennedy, Subharmonic Functions, vol. 1, Academic Press, 1976.

[7] M. Heins, Entire functions with bounded minimum modulus; subharmonic function analogues, Ann. Math. (2) 49 (1948), 200-213.

[8] A. Hinkkanen, On the modulus of continuity of analytic functions, Ann. Acad. Sci. Fenn. Ser. A I Math. 10 (1985), 247-253.

[9] A. Hinkkanen, On the majorization of analytic functions, Indiana Univ. Math. J. 36 (1987), 307-331.

[10] A. Hinkkanen, The sharp form of certain majorization theorems for analytic functions, Complex Variables Theory Appl. 12 (1989), 39-66.

[11] A. Hinkkanen, Majorisation of analytic functions, an extended abstract for an Oberwolfach meeting, 3 pages, in Normal families and complex dynamics, Abstracts from the workshop held February 18-24, 2007, pp. 487-547, Oberwolfach Reports. Vol. 4, no. 1, European Mathematical Society, Zürich, 2007.

[12] A. Hinkkanen, Majorization of analytic functions, Scientific Bulletin of Chełm, Section of Mathematics and Computer Science, 2 (2007), 25-30. 
[13] A. Hinkkanen, Majorization of the modulus of continuity of analytic functions, Comput. Methods Funct. Theory 8 (2008), No. 2, 303-325.

[14] Ch. Pommerenke, Univalent Functions, Vandenhoeck and Ruprecht, Göttingen, 1975.

[15] W. Smith and D. Stegenga, The local modulus of continuity of an analytic function, Holomorphic functions and moduli, Vol. I, pp. 133-142, Math. Sci. Res. Inst. Publ., 10, Springer, New York, 1988.

[16] P.M. Tamrazov, Contour and solid structural properties of holomorphic functions of a complex variable (Russian), Uspehi Mat. Nauk 28 (1973), 131-161. English translation with title "Contour and solid structure properties of holomorphic functions of a complex variable" in Russian Math. Surveys 28 (1973), 141-173.

[17] P.M. Tamrazov, Contour-solid properties of holomorphic functions and mappings with bilogarithmically concave majorants (Russian), Dokl. Akad. Nauk SSSR 270 (1983), 791-795. English translation in Soviet Math. Dokl. 27 (1983), 676-680.

[18] P.M. Tamrazov, Solid-contour results for holomorphic functions (Russian), Izv. Akad. Nauk SSSR Ser. Mat. 50 (1986), 835-848, 879. English translation with title "Contour-solid results for holomorphic functions" in Math. USSRIzvestia, 29 (1987), 193-205.

[19] P.M. Tamrazov, Sharpness of the fundamental bounds of contour-solid theorems (Russian), Ukrain. Mat. Zh. 41 (1989), 75-79, 135. English translation with title "Precision in the basic estimates in contour-solid theorems" in Ukrainian Math. J. 41 (1989), 68-72.

[20] P.M. Tamrazov, Finely holomorphic and finely subharmonic functions in contour-solid problems, Ann. Acad. Sci. Fenn. Ser. A I Math. 26 (2001), 325360 .

University of Illinois at Urbana-Champaign, Department of Mathematics, 1409 West Green Street, Urbana, IL 61801 USA

E-mail address: aimo@uiuc.edu 